\title{
Atopische Keratokonjunktivitis
}

\author{
Atopic Keratoconjunctivitis
}

Autoren

Institut

\author{
B. Sobolewska, M. Zierhut
}

Universitäts-Augenklinik, Eberhard-Karls Universität, Tübingen

\section{Schlüsselwörter \\ - atopische Kerato- konjunktivitis \\ - Keratoconjunctivitis vernalis \\ - Allergie \\ Key words \\ - atopic keratokonjunctivitis \\ - vernal keratoconjunctivitis \\ o allergy}

\section{Zusammenfassung \\ $\nabla$}

Es hat sich bewährt, die atopische Keratokonjunktivitis als eine nicht infektiöse entzündliche Augenerkrankung, die mit einer Atopie vergesellschaftet ist, zu definieren. Die Immunpathogenese wird bisher nicht vollständig verstanden. Die Diagnose basiert auf einer gezielten Anamnese und der klinischen Manifestation. Die Erkrankung kann einen überaus schwerwiegenden Verlauf nehmen. Daher benötigt ein erfolgreiches Management der atopischen Keratokonjunktivitis einen multidisziplinären Ansatz, welcher die Prävention, die dermatologische Betreuung und einen entsprechenden ophthalmologischen Behandlungsalgorithmus umfasst.

\section{Einleitung}

$\nabla$

eingereicht 5.3.2014

akzeptiert 13.3.2014

Bibliografie

DOI http://dx.doi.org/

10.1055/s-0034-1368396

Klin Monatsbl Augenheilkd

2014; 231: 512-517 @ Georg

Thieme Verlag KG Stuttgart .

New York · ISSN 0023-2165

Korrespondenzadresse

Dr. Bianka Sobolewska

Eberhard-Karls Universität

Universitäts-Augenklinik

Schleichstraße 12

72076 Tübingen

Tel.: + 49/(0)7071/2984008

Fax: + 49/(0)7071/294762

bianka.sob@gmx.de

\section{License terms}

\section{(1) $\Theta \circledast$}

Die atopische Keratokonjunktivitis (AKC) gehört neben der Keratoconjunctivitis vernalis zu den schwerwiegenden allergischen Erkrankungen des Auges, weil sie zu Hornhautkomplikationen führen und damit bedrohlich für die Sehschärfe sein kann [1,2], Beide Krankheiten unterscheiden sich von der allergischen saisonalen Konjunktivitis sowohl klinisch als auch pathophysiologisch [3].

Es hat sich für uns bewährt, die AKC abhängig von der Grunderkrankung der „Atopie“ zu definieren, wobei meistens eine atopische Dermatitis vorliegt, was aber unabhängig von Dauer und derzeitigem Grad der Atopie ist [4-6]. Die AKC findet man bei $25-40 \%$ der Patienten mit atopischer Dermatitis [7]. Die atopische Dermatitis ist eine chronisch entzündliche Hauterkrankung, die meist in der frühen Kindheit beginnt (zu $85 \%$ vor dem 5. Jahr) und bis in das Erwachsenenalter persistieren kann (70\% verlieren dann ihre Symptome) $[8,9]$. Erste Zeichen stellen gelblich-weiße, festhaftende Krusten bei Säuglingen dar, die als

\section{Abstract \\ $\nabla$}

It is useful to define atopic keratoconjunctivitis (AKC) as a non-infectious inflammatory condition of the ocular surface, which is associated with atopy. The pathogenesis of the disorder is not completely understood. The diagnosis is based on the patient's history and the clinical manifestations. Successful management of AKC, which can become a serious condition, requires a multidisciplinary approach that involves prevention, dermatological care and an adequate ophthalmological treatment algorithm.

Milchschorf bezeichnet werden. Bei Kindern und Erwachsenen treten typische Beugeekzeme in den Ellenbeugen-, Kniekehlen- und Handgelenken auf [8-10]. Diagnostisch hilfreich können auch charakteristische Atopiestigmata sein, wie bspw. eine tiefe Furchung der Handlinien, die auch „Ichthyosis- oder I-Hand“ genannt wird, eine doppelte Unterlidfalte nach Dennie-Morgan, eine Ausdünnung der Augenbrauen im seitlichen Bereich (Herthoge-Zeichen) und ein weißer Dermografismus [3]. Diagnostisch ist ein stark juckendes Ekzem in typischer Lokalisation entscheidend, aber auch der Krankheitsbeginn und das Vorliegen anderer Krankheiten aus dem atopischen Formenkreis, wie der allergischen Rhinitis und des allergischen Asthmas [8-10], können der Sicherung der Diagnose dienen.

Die Inzidenz der atopischen Dermatitis hat in den letzten 30 Jahren zugenommen und scheint noch weiter zuzunehmen. Derzeit wird geschätzt, dass in den Industrieländern etwa $10-20 \%$ aller Kinder und ca. 1-3\% der Erwachsenen von dieser Erkrankung betroffen sind [11]. 


\section{Ätiologie und Pathogenese \\ $\nabla$}

Ähnlich wie bei der atopischen Dermatitis liegt bei einer AKC eine Th2-dominierte Immunantwort vor. Nach Allergenkontakt setzen Th2-Zellen Interleukin-4 und Interleukin-5 (eosinophil chemoattractant) frei [12-14]. Diese konnten im Tränenfilm bei Patienten mit atopischer Keratokonjunktivitis in hohen Konzentrationen nachgewiesen werden [14]. Interleukin-4 stimuliert B-Zellen und führt zu einem Klassenswitch hin zu IgE-produzierenden B-Zellen [15]. Interleukin-5 wirkt positiv chemotaktisch auf eosinophile Granulozyten, deren Produkte wie IL-8 (neutrophil chemoattractant), ECP (eosinophilic cationic protein), und Eoxatin-1 und Eoxatin-2 die Entzündung weiter verstärken [6, 15-17]. Im weiteren Verlauf der chronischen Entzündung spielen auch proinflammatorische Interferon-gamma produzierende Th1-Zellen eine wichtige Rolle $[12,13]$.

\section{Klinik}

\section{$\nabla$}

Die AKC ist bilateral, jedoch oft asymmetrisch, und verläuft meistens ganzjährig. Saisonale Exazerbationen können jedoch vorkommen $[18,19]$. Die okuläre Symptomatik umfasst den überaus typischen Juckreiz, Fremdkörpergefühl, Schmerzen, Epiphora, Photophobie und gelegentlich ein verschwommenes Sehen. Typisch sind Lidveränderungen im Sinne einer Blepharitis mit Verkrustungen, Falten- und Rhagadenbildung. Das klinische Bild imponiert durch eine milde bis schwere Bindehautinjektion, Chemosis ( $\bigcirc$ Abb. 1) und eine papilläre Bindehautreaktion ( $\bigcirc$ Abb. 2) [20]. Die papilläre Hypertrophie oder pflastersteinartige Riesenpapillen $(>1 \mathrm{~mm}$ ) der Conjunctiva tarsi kommen gelegentlich mit fibrinöser Pseudomembranbildung vor. Dies führt nicht selten zu subepithelialen Bindehautnarben, dadurch dann zu Fornixverkürzung und nachfolgender Symblepharonbildung ( $\triangle$ Abb. 3) [18]. Diagnostisch sehr wichtig sind die typischen Trantas Dots (weißlich-gelbliche erhabene Ansammlungen eosinophiler und neutrophiler Granulozyten) ( $\boldsymbol{O} \mathbf{A b b}$.4) [21], die überwiegend limbusnah auftreten. Wegen einer schweren Benetzungsstörung geht die AKC nahezu immer mit einer Keratitis superficialis einher [22].

Typische Hornhautkomplikationen stellen eine Keratitis filiformis, ein persistierender Hornhautdefekt und ein Hornhautulkus („Schild-Ulkus“ oder „Atopie-Plaque“) ( $\bullet$ Abb. 5) dar, selten eine bakterielle oder virale (z.B. herpetische Keratitis [० Abb. 6]) Superinfektion [23]. Nach Abheilung des Hornhautulkus kann es zur Ausbildung von Hornhautnarben und Hornhautneovaskularisationen kommen.

$\mathrm{Zu}$ den weiteren Komplikationen der AKC wird eine Cataracta complicata (am häufigsten Cataracta subcapsularis anterior) [24] und ein Keratokonus $[25,26]$ sowie eine Netzhautablösung [27] gezählt. Takahashi et al. haben angedeutet, dass anteriore vitreoretinale Traktionen höchstwahrscheinlich eine wichtige Rolle in der Pathogenese der Netzhautablösung spielen [28]. Ein erhöhtes Risiko wurde auch für die Entwicklung von Bindehautkarzinomen gefunden [29].

Calonge und Herreras haben eine Unterteilung in Schweregrade vorgestellt, die für den Einsatz in klinischen Studien anwendbar ist [6]. Diese ist abhängig von der Ausprägung der subjektiven Beschwerden und der klinischen Symptome, insbesondere von Veränderungen an:

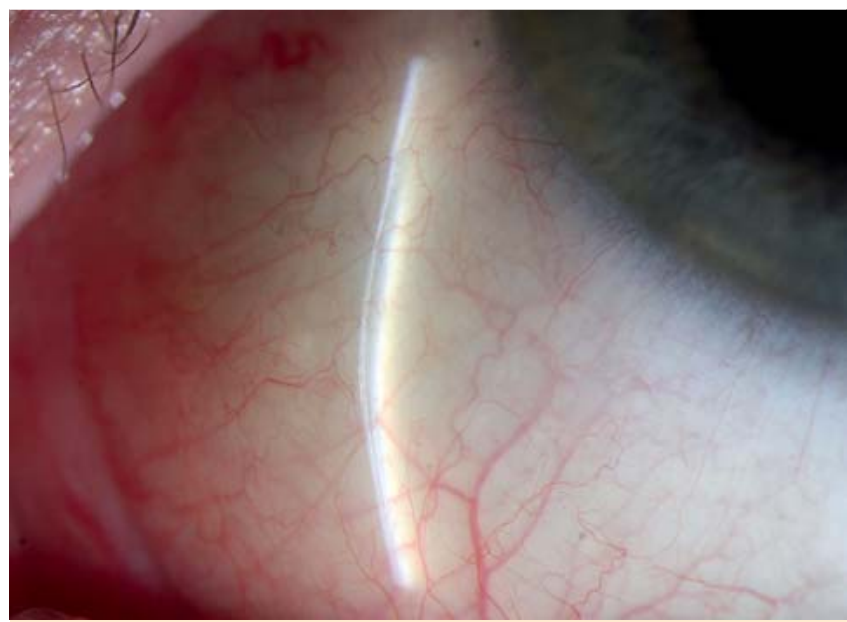

Abb. 1 Bindehaut-Chemosis bei akuter AKC.

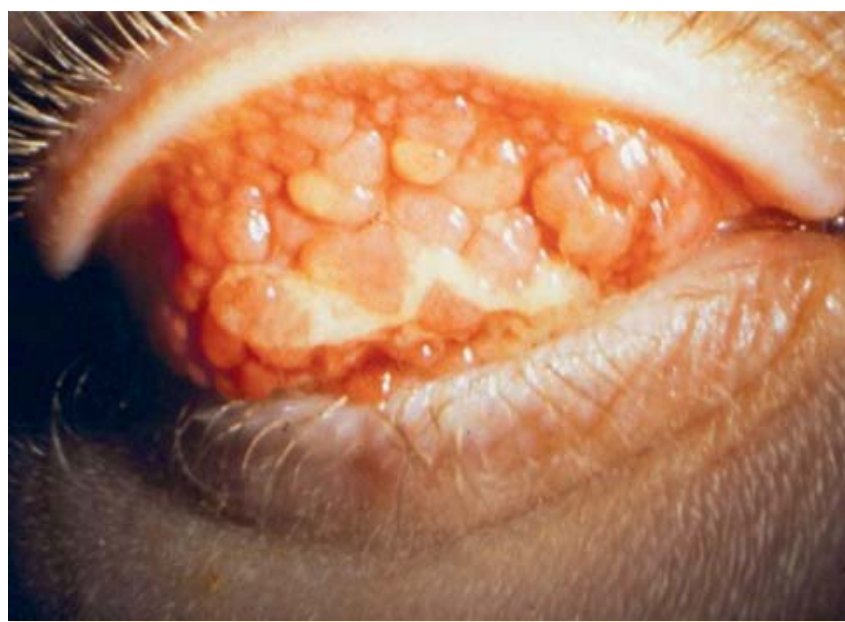

Abb. 2 Papilläre Reaktion der Conjunctiva tarsi bei AKC.

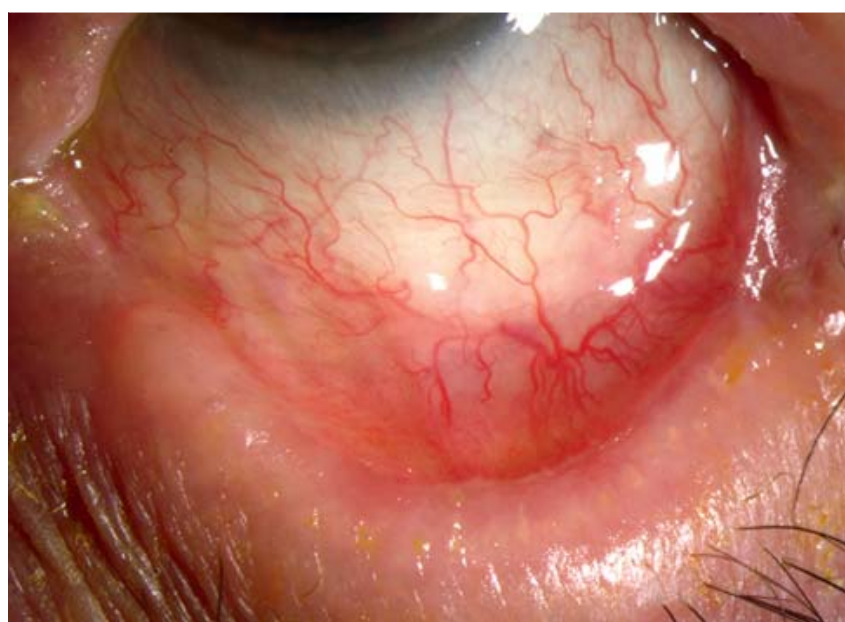

Abb. 3 Lidrandveränderungen mit Verdickung, Symblepharonbildung und Wimpernfehlstellung bei AKC. 
- Lidern (anteriore und/oder posteriore Blepharitis),

- Bindehaut (Papillenlokalisation: tarsal und/oder bulbär, vernarbende Bindehautveränderungen) und

- Hornhaut (Keratitis superficialis, persistierende oder nicht persistierende epitheliale Defekte, Hornhautneovaskularisationen).

\section{Diagnose \\ $\nabla$}

Die Diagnose basiert auf der Anamnese (positive Familienanamnese, Atopie) und dem klinischen Bild, wenn möglich dann auch mit der Identifizierung von Triggerfaktoren, wie Hausstaubmilben, Tierhaare, Pollen und Gräser. Das Vorliegen einer Typ-I Sensibilisierung kann mittels Hauttests (Prick-Testung, und AtopiePatch-Test) oder serologischer Untersuchungen (Gesamt und spezifisches Ig-E) verifiziert werden. Bei den meisten Patienten mit atopischer Dermatitis kann durch einen positiven Prick-Test das Vorliegen einer spezifischen Sensibilisierung gegen das entsprechende Allergen bestätigt werden (Leitlinie der Deutschen Dermatologischen Gesellschaft: „In vitro Allergiediagnostik“ http://www.awmf.org/leitlinien/detail/1l/061-017.html). Der Nachweis muss jedoch anschließend hinsichtlich seiner klinischen Relevanz überprüft werden [30].

Zum Monitoring des Schweregrads der AKC kann neben dem die Aktivität sehr gut widerspiegelnden klinischen Befund gemäß kürzlich publizierter Studien das ECP (eosinophil cationic protein) als Marker dienen. Sein Spiegel korreliert mit dem Schweregrad der atopischen Keratokonjunktivitis [31,32].

\section{Differenzialdiagnose \\ $\nabla$}

Differenzialdiagnostisch sollte v.a. die Keratoconjunctivitis vernalis (VKK) ausgeschlossen werden, die klinisch kaum von der AKC zu differenzieren ist. Diese tritt jedoch saisonal auf, und verläuft nicht so schwer wie die AKC. Laut Literatur sind die schwersten Verläufe von VKK bei Atopikern zu finden. Wir definieren für den klinischen Gebrauch daher die VKK so, dass wir Patienten mit einer Atopie einer „atopischen Keratokonjunktivitis“ zuordnen. Da die Erkrankung bei der AKK schwerer verläuft, können wir so die Therapie aggressiver gestalten, was bei der jahreszeitlich verlaufenden VKK oft nicht notwendig ist. So verlaufen z. B. die Hornhautneovaskularisationen bei der VKK meist weniger tief als bei der AKC [33].

Andere allergieinduzierte Augenerkrankungen wie die allergische Konjunktivitis, die gigantopapilläre Konjunktivitis und das allergische Kontaktekzem gehen typischerweise ohne Hornhautbeteiligungen einher.

Die allergische Konjunktivitis stellt die häufigste allergische Erkrankung des Auges dar und ist in eine saisonale Form (SAC seasonal allergic conjunctivitis; < 4 Wochen) und eine ganzjährige Form (PAC - perennial allergic conjunctivitis; > 4 Wochen) unterteilt [34]. Der Krankheitsverlauf der ganzjährigen allergischen Konjunktivitis ist jedoch mild, und papilläre Hypertrophie oder pflastersteinartige Riesenpapillen der Conjunctiva tarsi des oberen Lides treten selten auf [35], eine Hornhautbeteiligung fehlt. Für die Diagnose der gigantopapillären Konjunktivitis (GPC) und des allergischen Kontaktekzems spielt die Anamnese die entscheidende Rolle. Kontaktlinsen stellen die häufigste Ursache für die GPC dar, aber auch Augenprothesen, Fäden, Fremdkörper, Plomben und Sickerkissen können diese Krankheit hervorrufen.

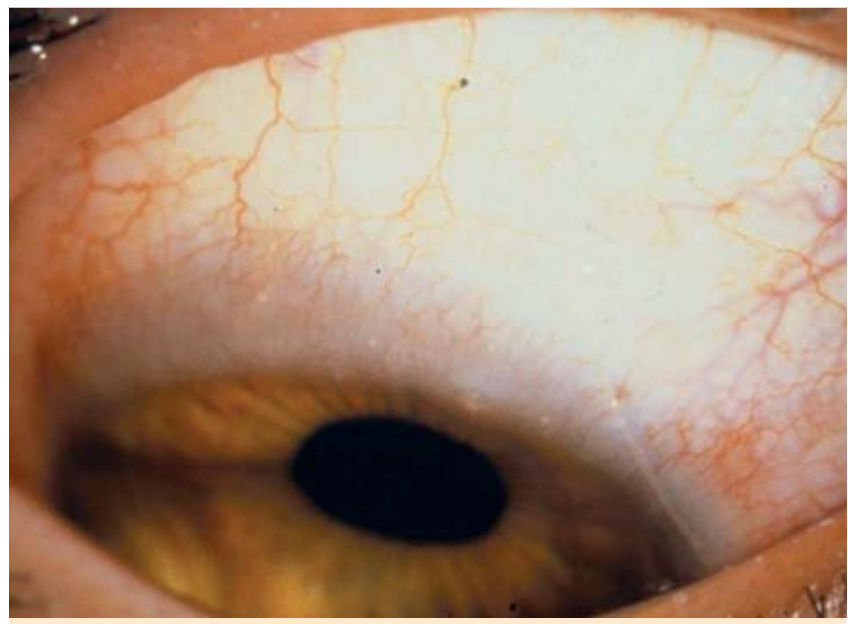

Abb. 4 Trantas Dots als Zeichen der akuten AKC.

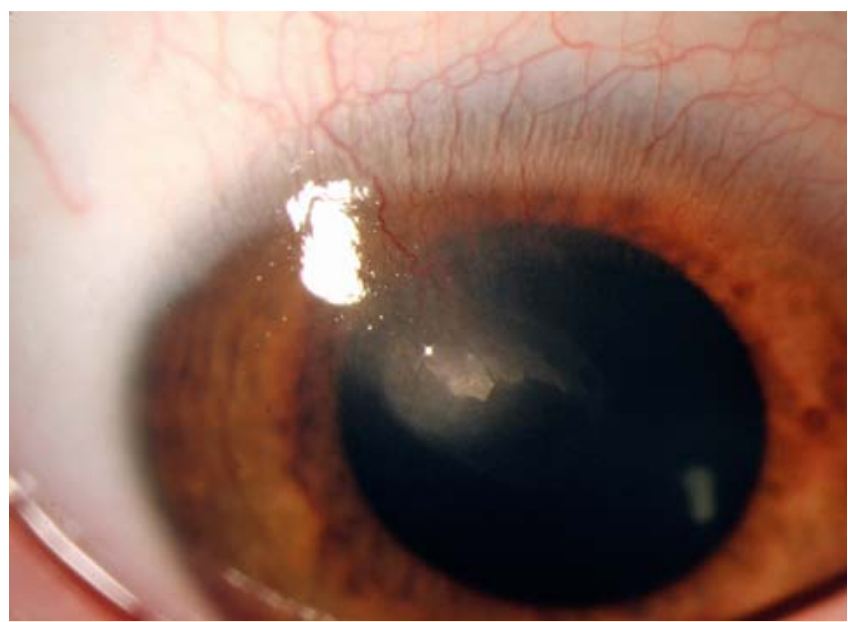

Abb. 5 Hornhautulkus mit Hornhautneovaskularisationen bei AKC.

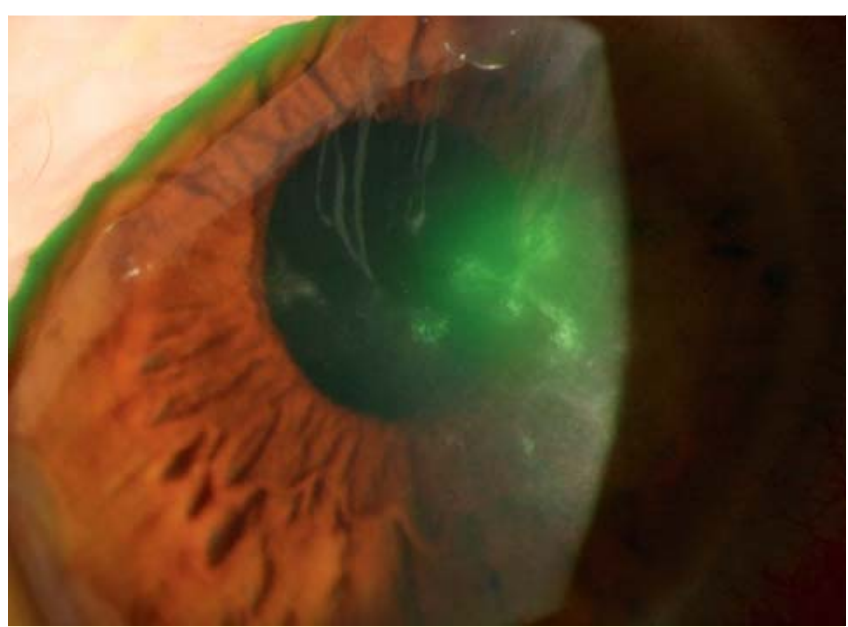

Abb. 6 Virale (HSV) Superinfektion bei AKC. 


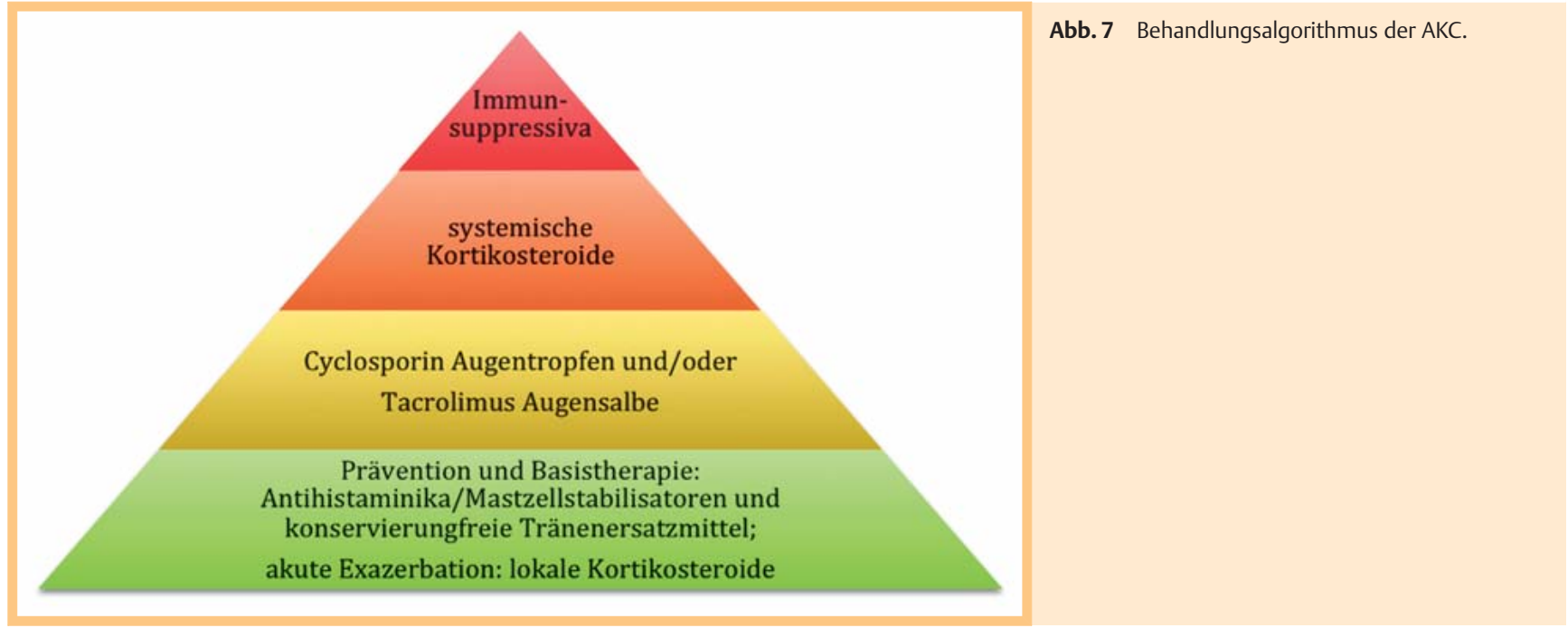

Die Kontaktallergie wird dagegen durch Tropfen, Salben, Verband oder Kosmetika ausgelöst. Therapeutisch führt eine Kontaktlinsenkarenz bei der GPC bzw. das Absetzen des induzierenden Antigens beim Kontaktekzem zur Verbesserung der Symptome und auch des Augenbefunds [36,37].

Andere Krankheiten, die differenzialdiagnostisch in Betracht gezogen werden sollten, sind

- Keratokonjunktivitis epidemica (asymmetrische, hochkontagiöse Konjunktivitis, beginnend mit Plica- und Karunkelschwellung, Lymphknotenvergrößerung und ggf. Bindehautmembranen, später oft nummuläre subepitheliale Hornhautinfiltrate),

- Keratitis marginalis (assoziiert mit Blepharitis, asymmetrisch vorkommende subepitheliale Hornhautinfiltrate),

- Keratokonjunktivitis bei Rosazea (die Prävalenzspitze liegt zwischen 50. und 60. Lebensjahr, eventuell auch typischer Hautbefund und Lidteleangiektasien) [38] und

- Keratitis herpetica (fast immer einseitig, eher milde Bindehautrötung, eventuell sogar anteriore Uveitis mit HornhautRückflächenbeschlägen).

- Im Falle unspezifischer Bindehautnarben oder eines Symblepharons sollte differenzialdiagnostisch besonders das okuläre Pemphigoid ausgeschlossen werden (durchschnittlicher Krankheitsbeginn liegt bei 65 Jahren, im Frühstadium tritt eine Fornixverkürzung auf, erst später bildet sich ein Symblepharon) [39].

\section{Therapie \\ $\nabla$}

Das Ziel der Therapie muss die Reduktion der subjektiven Symptomatik (u. a. Juckreiz, Fremdkörpergefühl, Epiphora, Schmerzen) und eine Verbesserung des Augenbefunds, wie Keratitis superficialis, Bindehautinjektion, subtarsale Papillen, Trantas Dots und v.a. des Hornhautulkus, sein. A Abb. 7 stellt unseren Behandlungsalgorithmus für die AKC vor.

Eine Basistherapie führen wir bei allen Formen der AKC durch, wobei die Konsequenz, mit der sie empfohlen wird, von dem bisherigen Krankheitsverlauf abhängig ist. Je mehr Kortikosteroide während des letzten Rezidivs benötigt wurden, desto konsequenter wird die Basistherapie empfohlen, unabhängig davon, ob die Erkrankung jahreszeitlich begrenzt oder ganzjährig ab- läuft. Die Basistherapie besteht aus Antihistaminika/Mastzellstabilisatoren (Olopatadin, Ketotifenfumarat, Epinastin) und intensiver Therapie mit konservierungsmittelfreien Tränenersatzmitteln. Auch die Anwendung von Cyclosporin A (CsA) AT gehört hierzu. Die bisher vorgestellten Daten weisen darauf hin, dass die ergänzende Applikation von CsA einen steroidsparenden Effekt hat. Darüber hinaus zeigten die CsA-Augentropfen eine positive Wirkung auf die Behandlung des Hornhautulkus [2,40]. Bei steroid- und CsA-resistenten oder bei steroidabhängigen Patienten kann auch Tacrolimus (Calcineurin-Inhibitor) eingesetzt werden, welches eine gute Wirksamkeit bei Patienten mit der AKC gezeigt hat [41-43]. Bei Patienten mit einer ausgeprägten atopischen Blepharitis wurde auch eine Kombination von CsA-Augentropfen und Tacrolimus-Salbe vorgeschlagen. Dies kann möglicherweise einen additiven Effekt ausüben [44].

Der akute Schub (charakterisiert gelegentlich durch BindehautChemosis, v.a. aber durch das Auftreten von Trantas Dots), erfordert nahezu immer zumindest den limitierten Einsatz von lokalen Kortikosteroiden. Hier favorisieren wir Loteprednolol bzw. Rimexolon wegen des besseren Nebenwirkungsprofils im Gegensatz zu Prednisolon, Dexamethason und Bethametason. Im Falle von häufigen Schüben oder moderaten bis schweren Verlaufsformen, die eine langfrisitige Gabe von Kortikosteroiden erfordern, und damit zu kortikosteroidbedingten Komplikationen (Katarakt, Glaukom) führen könnten, hat sich der Einsatz von CsA bewährt.

Bei Versagen der lokalen Therapie, v. a. aber beim Vorliegen eines Hornhautulkus, sind systemische Kortikosteroide Mittel der Wahl $[12,30]$. Um systemische Steroide zu sparen, sollten systemische Immunsuppressiva wie Cyclosporin [30,45], Azathioprin, Methotrexat, Mycophenolatmofetil $[30,46,47]$ oder Tacrolimus [48] in schweren Fällen, eingesetzt werden.

Beim Hornhautulkus, das trotz der kombinierten lokalen Therapie und der systemischen Gabe von Kortikosteroiden nicht heilt, stellt eine Amnionmembranaufnähung eine weitere Therapieoption dar [49].

Topische Vasokonstriktoren sollten aufgrund der Nebenwirkungen (Rebound-Hyperämie und gelegentlich Mydriasis) und der fehlenden Wirksamkeit auf die allergische Reaktion (im Vergleich zu Mastzellstabilisatoren/Antihistaminika) keinesfalls verwendet werden. 


\section{Prävention}

$\nabla$

Im Rahmen einer Prävention spielen Allergenkarenz und Milieusanierung eine bedeutende Rolle in der Therapie der AKC. Als Maßnahme zur Prävention wird die Reduktion der Exposition gegenüber Hausstaubmilbenallergenen empfohlen, z. B. die Anwendung von hypoallergener Bettwäsche, das Vermeiden von Teppichen etc. Die Haustierhaltung sollte eingeschränkt werden. Ein Innenraumklima, das Schimmelpilzwachstum begünstigt (hohe Luftfeuchtigkeit, mangelnde Ventilation), sollte unbedingt vermieden werden (Leitlinie der Deutschen Dermatologischen Gesellschaft: „Allergieprävention“ http://www.awmf.org/leitlinien/ detail/1l/061-016.html). Des Weiteren ist eine Sonnenbrille zum Schutz vor Allergenexposition und konservierungsmittelfreie Tränenersatzmittel zum Ausspülen des Auges nach Antigenkontakt, aber auch zur Prävention des trockenen Auges, zu empfehlen. Der jährliche Start lokaler Antihistaminika/Mastzellstabilisatoren sollte bei bekannter allergischer Reaktion unbedingt frühzeitig erfolgen (z.B. 4 Wochen vor Beginn der Allergiezeit).

\section{Zusammenfassung \\ $\nabla$}

Bei der AKC kommt es oft aufgrund der sehr ähnlichen klinischen Symptomatik und auch des überlappenden Krankheitsverlaufs (saisonale Exazerbation) zu Abgrenzungsschwierigkeiten mit der VKK, was man umgehen kann, wenn man die AKC abhängig von einer bestehenden Atopie definiert. Darüber hinaus kann der Augenbefund nicht nur anderen Allergieerkrankungen, sondern auch anderen Erkrankungen wie einer Rosazea oder einem okulären Pemphigoid ähneln. Daher empfiehlt sich bei Verdacht auf eine allergische Augenerkrankung stets eine dermatologische Konsultation, um eine zugrunde liegende Hautkrankheit einzuordnen. In Zusammenarbeit mit dem Dermatologen kann auch die systemische Therapie im Falle von häufigen Exazerbationen oder bei schwerem Krankheitsverlauf diskutiert werden.

Ein anderes Problem bei Patienten mit der AKC stellt das gefürchtete Hornhautulkus dar, welches trotz systemischer Therapie nicht ausreichend abheilt. Hier sollte eine Amnionmembranaufnähung in Erwägung erzogen werden. Die AKC stellt auch heute noch eine große Herausforderung dar, die leider oft fehldiagnostiziert und auch falsch behandelt wird.

\section{Interessenkonflikt}

$\nabla$

Nein.

\section{Literatur}

1 Power WJ, Tugal-Tutkun I, Foster CS. Long-term follow-up of patients with atopic keratoconjunctivitis. Ophthalmology 1998; 105: 637-642

2 Tomida I, Schlote T, Bräuning J et al. Cyclosporin A 2\% eyedrops in therapy of atopic and vernal keratoconjunctivitis. Ophthalmologe 2002; 99: 761-767

3 Hanifin JM. Atopiform dermatitis: do we need another confusing name for atopic dermatitis? Br J Dermatol 2002; 147: 430-432

4 Hogan MJ. Atopic keratoconjunctivitis. Trans Am Ophthalmol Soc 1952; 50: $265-281$

5 Guglielmetti S, Dart JK, Calder V. Atopic keratoconjunctivitis and atopic dermatitis. Curr Opin Allergy Clin Immunol 2010; 10: 478-485

6 Calonge $M$, Herreras JM. Clinical grading of atopic keratoconjunctivitis. Curr Opin Allergy Clin Immunol 2007; 7: 442-445

7 Foster CS, Calonge M. Atopic keratoconjunctivitis. Ophthalmology 1990; 97: 992-1000

8 Leung DY, Bieber T. Atopic dermatitis. Lancet 2003; 361: 151-160
9 Bieber T. Mechanisms of disease: atopic dermatitis. N Engl J Med 2008; 358: 1483-1494

10 Watson W, Kapur S. Atopic dermatitis. Allergy Asthma Clin Immunol 2011; 7 (Suppl. 1): S4

11 Larsen FS, Hanifin JM. Epidemiology of atopic dermatitis. Immunol Allergy Clin North Am 2002; 22: 1-25

12 Novak N, Bieber T. Pathophysiologie der atopischen Dermatitis: Neue Erkenntnisse und der Nutzen für die Praxis. Dtsch Arztebl 2004; 101 : A-108/B-94/C-92

13 Leonardi A, Fregona IA, Plebani M et al. Th1- and Th2-type cytokines in chronic ocular allergy. Graefes Arch Clin Exp Ophthalmol 2006; 244: $1240-1245$

14 Uchio E, Ono SY, Ikezawa Z et al. Tear levels of interferon-gamma, interleukin (IL) -2, IL-4 and IL-5 in patients with vernal keratoconjunctivitis, atopic keratoconjunctivitis and allergic conjunctivitis. Clin Exp Allergy 2000; 30: 103-139

15 Lampinen M, Carlson M, Håkansson LD et al. Cytokine-regulated accumulation of eosinophils in inflammatory disease. Allergy 2004; 59: 793-805

16 Montan PG, van Hage-Hamsten M. Eosinophil cationic protein in tears in allergic conjunctivitis. $\mathrm{Br} J$ Ophthalmol 1996; 80: 556-560

17 Leonardi A, Jose PJ, Zhan H et al. Tear and mucus eotaxin-1 and eotaxin2 in allergic keratoconjunctivitis. Ophthalmology 2003; 110: 487-492

18 Brewitt H, Zierhut M. Trockenes Auge: Anatomie, Physiologie, Pathophysiologie, Diagnostik, Therapie. Heidelberg: Kaden; 2001

19 Rachdan D, Anijeet DR, Shah S. Atopic keratoconjunctivitis: present day diagnosis. Br J Ophthalmol 2012; 96: 1361-1362

20 Bielory B, Bielory L. Atopic dermatitis and keratoconjunctivitis. Immunol Allergy Clin North Am 2010; 30: 323-336

21 Sy H, Bielory L. Atopic keratoconjunctivitis. Allergy Asthma Proc 2013; 34: 33-41

22 Tuft SJ, Kemeny DM, Dart JK et al. Clinical features of atopic keratoconjunctivitis. Ophthalmology 1991; 98: 150-158

23 Easty D, Entwistle C, Funk A et al. Herpes simplex keratitis and keratoconus in the atopic patient. A clinical and immunological study. Trans Ophthalmol Soc U K 1975; 95: 267-276

24 Bair B, Dodd J, Heidelberg K et al. Cataracts in atopic dermatitis: a case presentation and review of the literature. Arch Dermatol 2011; 147: 585-588

25 Bawazeer AM, Hodge WG, Lorimer B. Atopy and keratoconus: a multivariate analysis. Br J Ophthalmol 2000; 84: 834-836

26 Rahi A, Davies P, Ruben $M$ et al. Keratoconus and coexisting atopic disease. Br J Ophthalmol 1977; 61: 761-764

27 Yoneda K, Okamoto $H$, Wada Y et al. Atopic retinal detachment. Report of four cases and a review of the literature. Br J Dermatol 1995; 133 : 586-591

28 Takahashi M, Suzuma K, Inaba I et al. Retinal detachment associated with atopic dermatitis. Br J Ophthalmol 1996; 80: 54-57

29 Kallen C, Reinhard T, Schilgen G et al. [Atopic keratoconjunctivitis: probably a risk factor for the development of conjuntival carcinoma]. Ophthalmologe 2003; 100: 808-814

30 Akdis CA, Akdis M, Bieber T et al.; European Academy of Allergology and Clinical Immunology/American Academy of Allergy Asthma and Immunology. Diagnosis and treatment of atopic dermatitis in children and adults: European Academy of Allergology and Clinical Immunology/ American Academy of Allergy, Asthma and Immunology/PRACTALL Consensus Report. J Allergy Clin Immunol 2006; 118: 152-169

31 Wakamatsu TH, Satake Y, Igarashi A et al. IgE and eosinophil cationic protein (ECP) as markers of severity in the diagnosis of atopic keratoconjunctivitis. Br J Ophthalmol 2012; 96: 581-586

32 Wakamatsu TH, Tanaka M, Satake Y et al. Eosinophilic cationic protein as a marker for assessing the efficacy of tacrolimus ophthalmic solution in the treatment of atopic kertaoconjunctivitis. Mol Vis 2011; 17: 932-938

33 Jun J, Bielory L, Raizman MB. Vernal conjunctivitis. Immunol Allergy Clin North Am 2008; 28: 59-82

34 O'Brien TP. Allergic conjunctivitis: an update on diagnosis and management. Curr Opin Allergy Clin Immunol 2013; 13: 543-549

35 Bielory L. Ocular allergy overview. Immunol Allergy Clin North Am 2008; 28: 1-23

36 Kari O, Saari KM. Updates in the treatment of ocular allergies. J Asthma Allergy 2010; 3: 149-158

37 Ehlers WH, Donshik PC. Giant papillary conjunctivitis. Curr Opin Allergy Clin Immunol 2008; 8: 445-449

38 Sobolewska B, Zierhut M. Ocular rosacea. Hautarzt 2013; 64: 506-508 
39 Sobolewska B, Deuter C, Zierhut M. Current medical treatment of ocular mucous membrane pemphigoid. Ocul Surf 2013; 11: 259-266

40 González-López JJ, López-Alcalde J, Morcillo Laiz R et al. Topical cyclosporine for atopic keratoconjunctivitis. Cochrane Database Syst Rev 2012; 9: CD009078

41 Sakarya $Y$, Sakarya R. Treatment of refractory atopic blepharoconjunctivitis with topical tacrolimus $0.03 \%$ dermatologic ointment. J Ocul Pharmacol Ther 2012; 28: 94-96

42 García DP, Alperte JI, Cristóbal JA et al. Topical tacrolimus ointment for treatment of intractable atopic keratoconjunctivitis: a case report and review of the literature. Cornea 2011; 30: 462-465

43 Al-Amri AM. Long-term follow-up of tacrolimus ointment for treatment of atopic keratoconjunctivitis. Am J Ophthalmol 2014; 157: 280-286
44 Tzu JH, Utine CA, Stern ME et al. Topical calcineurin inhibitors in the treatment of steroid-dependent atopic keratoconjunctivitis. Cornea 2012; 31: 649-654

45 Cornish KS, Gregory ME, Ramaesh K. Systemic cyclosporin A in severe atopic keratoconjunctivitis. Eur J Ophthalmol 2010; 20: 844-851

46 Akhavan A, Rudikoff D. Atopic dermatitis: systemic immunosuppressive therapy. Semin Cutan Med Surg 2008; 27: 151-155

47 BuBmann C, Bieber T, Novak N. Systemic therapeutic options for severe atopic dermatitis. J Dtsch Dermatol Ges 2009; 7: 205-219

48 Stumpf T, Luqmani N, Sumich P et al. Systemic tacrolimus in the treatment of severe atopic keratoconjunctivitis. Cornea 2006; 25: 11471149

49 Takano Y, Fukagawa K, Miyake-Kashima M et al. Dramatic healing of an allergic corneal ulcer persistent for 6 months by amniotic membrane patching in a patient with atopic keratoconjunctivitis: a case report. Cornea 2004; $23: 723-725$ 